\title{
Structure and Evolution of Planetary Nebula Haloes
}

\author{
Matthias Steffen \& Detlef Schönberner \\ Astrophysikalisches Institut Potsdam, Germany
}

\begin{abstract}
The density structure of the extended haloes of Planetary Nebulae (PN) is generally believed to reflect the previous history of heavy mass loss during the final stages of stellar evolution on the asymptotic giant-branch (AGB). In this review, we discuss different interpretations of the observed $\mathrm{PN}$ halo structures in the light of recent numerical simulations combining detailed AGB and post-AGB stellar evolution calculations with time-dependent hydrodynamical wind models.
\end{abstract}

\section{Introduction}

Faint extended haloes around planetary nebulae (PNe) are a quite common phenomenon, being found in about $70 \%$ of the elliptical PNe for which adequately deep images exist (cf. Corradi, this volume). Although the existence of PN haloes is known for a long time (e.g. the giant halo of NGC 6826 discovered by Duncan 1937), their low surface brightness and neighborhood to the bright central structures have made it difficult to obtain reliable spectrophotometric data which can serve as a basis to constrain theories of their nature and origin. It is generally accepted that PN haloes are the relic of the slow wind from the $\mathrm{PN}$ precursor in its AGB phase of evolution into which the wind from the PN central star is running with higher speed. However, there is still some confusion about the relationship between the occurrence of distinct mass loss episodes on the AGB, and the internal structure of Planetary Nebulae including their haloes.

\section{Different types of haloes and their astrophysical interpretation}

\subsection{Double-shell nebulae}

Many of the round or elliptical PNe show a so-called double-shell structure (e.g. IC 3568, NGC 6578): the inner region, enclosed by a thin bright rim is embedded in a fainter nebulosity called an 'attached' shell by Chu et al. (1987) and an inner halo by Balick (1987). When the understanding of PNe gas dynamics was still fairly undeveloped, the occurrence of the double shell structure was thought to be the result of two distinct mass ejections (e.g. Trimble \& Sackmann 1978). It is now known from detailed hydrodynamical modeling (Marten \& Schönberner 1991, Frank 1994, Mellema 1994) that photo-ionization together with two-wind interaction inevitably leads to the formation of these typical double-shell structures, where the (outer) shell, driven by the thermal pressure of ionized AGB material, is fainter than the rim, which is the result of compression 
of the inner nebula by the pressure of the hot bubble (cf. Schönberner \& Steffen, this volume). We may therefore conclude that the whole PN structure has no relation to any mass loss events. Nevertheless, even some recent investigations still prefer a different interpretation, relating the outer edge of a double-shell nebula to a thermal pulse (e.g. Hajian et al. 1997).

\subsection{Multiple-shell nebulae and haloes}

In line with the terminology of Chu et al. (1987), we henceforth use the term halo only for outer limb-brightened ('detached') structures that have a surface brightness $I_{\text {halo }} \lesssim 0.01 I_{\max }$, and a size at least twice as large as the main shell $\left(r_{\text {halo }} \gtrsim 2 r_{\text {shell }}\right)$. The 'attached' shells described above cannot be haloes according to this definition, since they are much brighter $I_{\text {shell }} \gtrsim 0.1 I_{\max }$, and generally have a smaller relative size $\left(r_{\text {shell }} \lesssim 2 r_{\text {rim }}\right)$.

Even with this more restrictive definition, one still has to distinguish between two physically different kind of haloes. An instructive example is the well-studied, old PN NGC 2438. Here a bright central 'core' is surrounded by a relatively bright 'halo'. Recently, the existence of a second limb-brightened halo was revealed in very deep images by Rauch et al. (1999) and Corradi et al. (2000). According to the detailed investigation of the latter authors, the observational facts together with the results from hydrodynamical modeling and stellar evolution calculations provide convincing evidence that the inner halo is actually the recombined shell of what was formerly a typical double-shell configuration. Such recombination haloes may develop when recombination sets in as a consequence of the fast luminosity drop of the PN central star during the advanced post-AGB evolution (Tylenda 1986). In fact, the luminosity of the central star of NGC 2438 is rather low, $L<500 L_{\odot}$. If this interpretation is correct, then neither the outer edge of the recombination halo nor the edge of the central 'core' is related to thermal pulses on the AGB, as required for the application of the timescale-correlation method by Hajian et al. (1997).

The existence of the faint outer halo is naturally explained within the standard scheme of AGB stellar evolution as well: it probably consist of photoionized AGB matter whose density structure still preserves information about the mass loss history during the last thermal pulse cycle on the asymptotic giant branch. These genuine $A G B$ haloes are therefore a suitable subject of 'stellar wind paleontology' (see Balick et al. 1992).

A famous example of a PN with a well developed AGB halo is NGC 6826. This relatively young, typical double-shell nebula is embedded in a rather big halo $\left(r_{\text {halo }} \gtrsim 5 r_{\text {shell }}\right)$ of low surface brightness $\left(I_{\text {halo }} \lesssim 0.001 I_{\text {rim }}\right)$. Even though the inner nebula is of clearly elliptical shape, the AGB halo exhibits a remarkable spherical symmetry. The outer edge of the limb-brightened halo was proposed to be the result of the interaction of the AGB wind with the interstellar medium (Plait \& Soker 1990). We will argue below that the sharp outer edges of genuine $A G B$ haloes are more likely the signature of the final thermal pulse on the AGB.

\section{Structure and Dynamics of AGB wind envelopes}

All attempts to model the structure and evolution of PN haloes must start with a proper description of the final mass loss history on the AGB. 

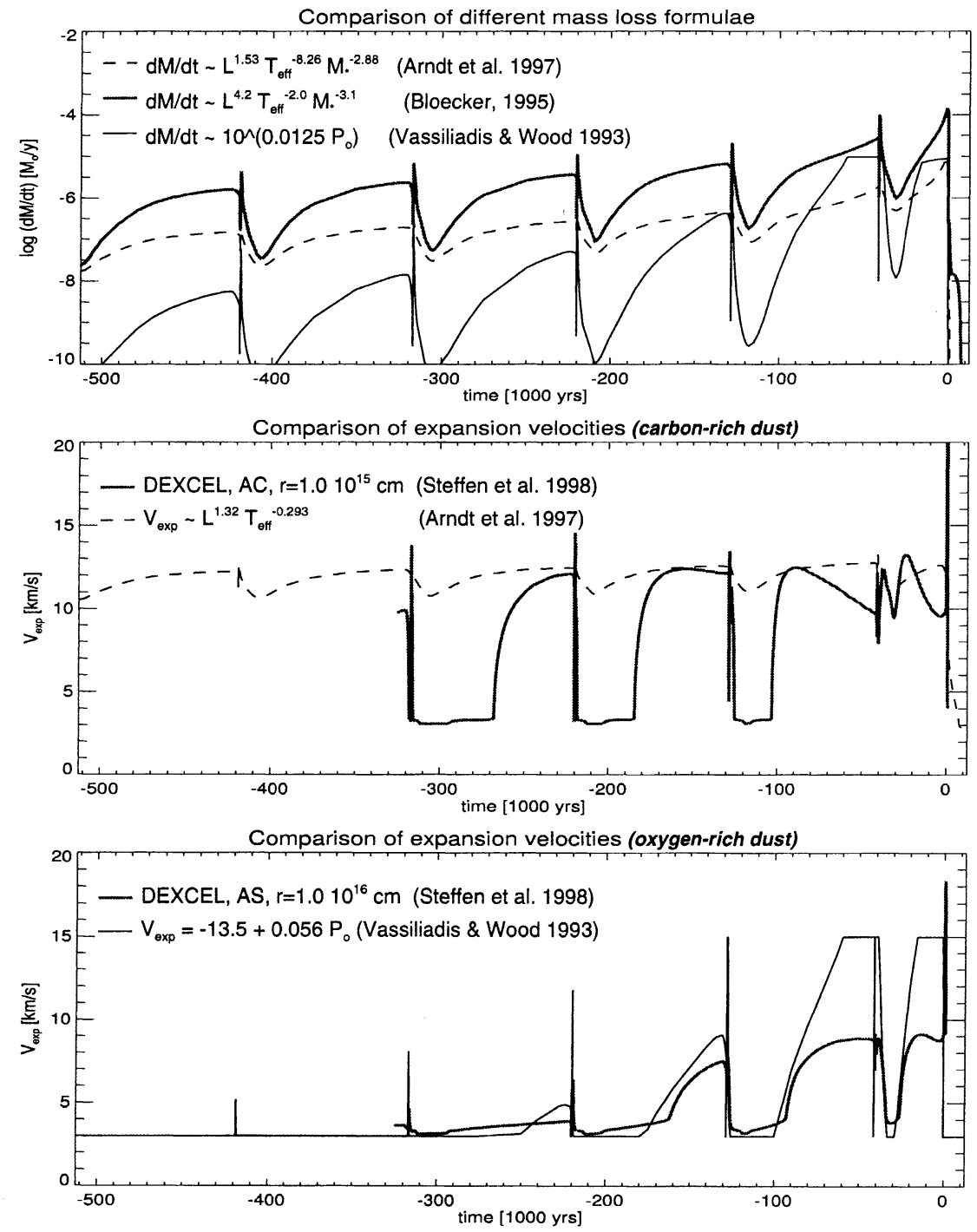

Figure 1. Different descriptions of AGB mass loss ( $\dot{M}$, top) and expansion velocity ( $V_{\exp }$, lower). The semi-empirical mass loss formula by Blöcker (1995) was used to compute the evolutionary track of a $3 \mathrm{M}_{\odot}$ star. The theoretical relations by Arndt et al. (1997), valid only for carbon-rich dust, and the empirical formulae by Vassiliadis \& Wood (1993) (with the fundamental mode pulsation period $\left.P_{0}[d]=8.510^{-3}\left(R / R_{\odot}\right)^{1.94}\left(M / M_{\odot}\right)^{-0.9}\right)$ were then applied to this given track to evaluate $\dot{M}$ and $V_{\text {exp }}$. The expansion velocities by Steffen et al. (1998) were obtained from two-fluid hydrodynamics, assuming amorphous carbon (middle) and silicate (bottom) dust, respectively. 
Unfortunately, fully self-consistent theoretical models of AGB mass loss are not yet available due to the enormous complexity of the involved physical processes, although considerable progress has already been made for carbon-rich AGB stars (e.g. Arndt et al. 1997; Winters et al. 2000). Up to now, calculations of AGB stellar evolution are based on empirical (e.g. Vassiliadis \& Wood 1993) or semi-empirical (e.g. Blöcker 1995) prescriptions of the mass loss rate as a function of stellar parameters. A comparison of different mass loss formulae is presented in Fig. 1. We note that all of these descriptions predict considerable variations of the mass-loss rate and outflow velocity during a thermal pulse cycle.

It is clear that these variations must lead to a corresponding modulation of the density in the circumstellar AGB wind envelope. But only gasdynamical simulations can give quantitative information about the density and velocity structure imposed on the AGB wind envelope by the changing stellar luminosity and mass loss rate. A first step into this direction has been reported by Vassiliadis \& Wood (1992). The most detailed model calculations of the long-term dynamical evolution of AGB wind envelopes are those by Steffen et al. (1998), who utilize a two-component radiation hydrodynamics code designed to model a stellar wind driven by radiation pressure on dust, together with the results of stellar evolution calculations by Blöcker (1995) as a time-dependent inner boundary condition.

The numerical simulations by Steffen et al. (1998) have shown that, not unexpectedly, the phases of low mass-loss rate produce distinct regions of low density ('gaps') within the circumstellar AGB envelope, often bounded by sharp edges where the density changes by up to two orders of magnitude. In addition, the two-wind interaction mechanism can work in AGB outflows as well, producing very thin shells of greatly enhanced gas density ${ }^{9}$, which have a long lifetime and propagate outward with the wind to considerable distances. According to Steffen \& Schönberner (2000, 2001), two distinct conditions give rise to two-wind interaction: (i) a mass loss 'eruption' as a result of a helium-shell flash, and (ii) a rapid increase of the outflow velocity after the stellar luminosity and mass loss rate exceed the critical limit for efficient wind acceleration by radiation pressure on dust. The compressed shells resulting from scenario (i) are located close to the outer edge of a 'gap'. Very likely, such shells can be identified with the geometrically thin $\mathrm{CO}$ shells discovered recently around some optically visible carbon-rich AGB stars like TT Cygni (Olofsson et al. 2000). The shells produced via scenario (ii) have roughly the same thickness and amplitude, but tend to sit at the inner edge of a 'gap'. They have not (yet) been observed in AGB winds, but we suggest that they are responsible for the limb-brightening of observed AGB haloes (see below). A broad 'gap' with compressed shells at both edges is clearly seen in Fig. 2 (top) around $r=10^{18} \mathrm{~cm}$.

As demonstrated in Fig. 2, the radial density structure of the circumstellar wind envelope at the end of the AGB depends not only on the previous mass-loss history $\dot{M}(t)$, but also on the magnitude and variability of the outflow velocity

\footnotetext{
${ }^{9}$ These shells must not be confused with the concentric arc-like structures found around some proto-planetary nebulae like CRL 2688 or the AGB object IRC +10216 , which are ejected on much shorter time scales and are restricted to the inner regions of the circumstellar environment (cf. Simis et al. 2001 for a theoretical interpretation of this phenomenon).
} 

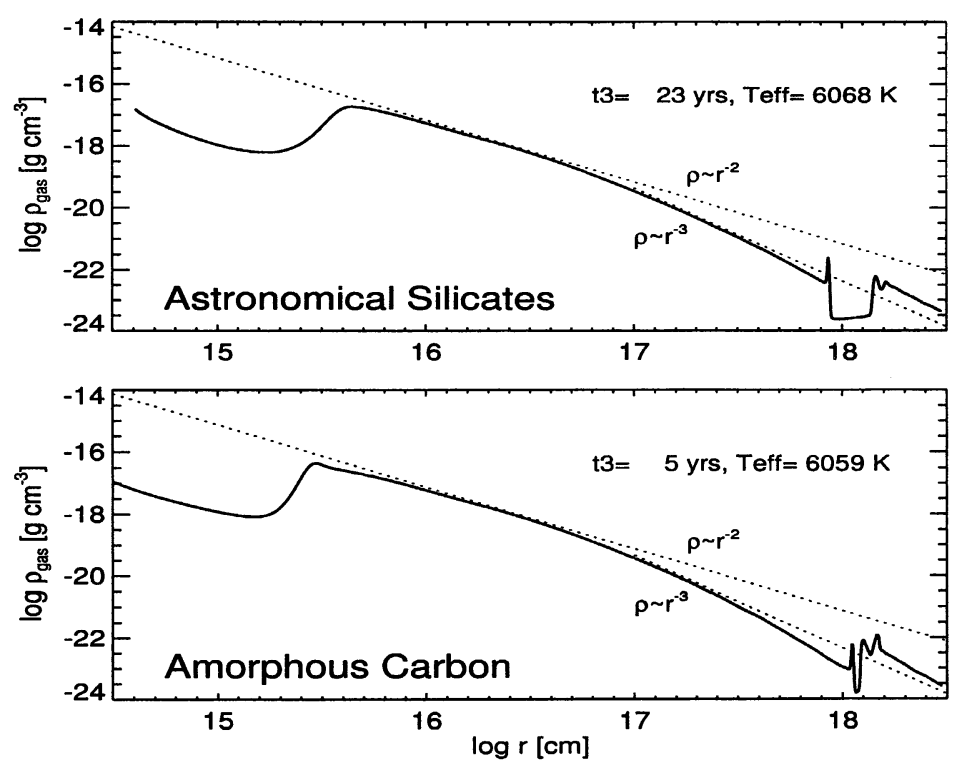

Figure 2. Radial density distribution of the circumstellar wind envelope at the end of the AGB, obtained from two-component hydrodynamical simulations (cf. Steffen \& Schönberner 2000), assuming silicate based (top) and carbon based (bottom) dust chemistry, respectively. In both cases, the adopted stellar evolution and mass loss history are identical (see upper curve in the top panel of Fig. 1).

$V_{\exp }(t)$. The latter is different for carbon and oxygen based dust, respectively (cf. Fig. 1), due to the different radiative absorption efficiency of the two type of dust grains. Note that, as a consequence of the adopted mass loss prescription ( $\dot{M}$ increasing towards the end), the density profile in the main part of the final AGB wind envelope falls off as $\rho \sim r^{-3}$ or even steeper in both cases.

\section{Modeling the evolution of PN haloes}

Most of the hydrodynamical simulations of the evolution of planetary nebulae start from simple initial conditions, typically assuming $\rho \sim r^{-\alpha}(\alpha=2$ or 3; e.g. Mellema 1994), thus ignoring the previous mass loss variations on the AGB and the influence of the interstellar medium (see however Villaver et al., this volume). Such modeling is obviously inappropriate to study the structure and evolution of PN haloes. In contrast, the PN models of Schönberner et al. (1997) start out with realistic initial configurations like those seen in Fig. 2. Apart from the restriction to spherical symmetry, these models account for all essential physical processes driving the formation and evolution of a PN and its halo: post-AGB stellar evolution with mass loss (Blöcker 1995) and a realistic wind model based on the theory of Pauldrach et al. (1988) acting as an inner boundary condition for hydrodynamical simulations, coupled with a time-dependent treatment of 

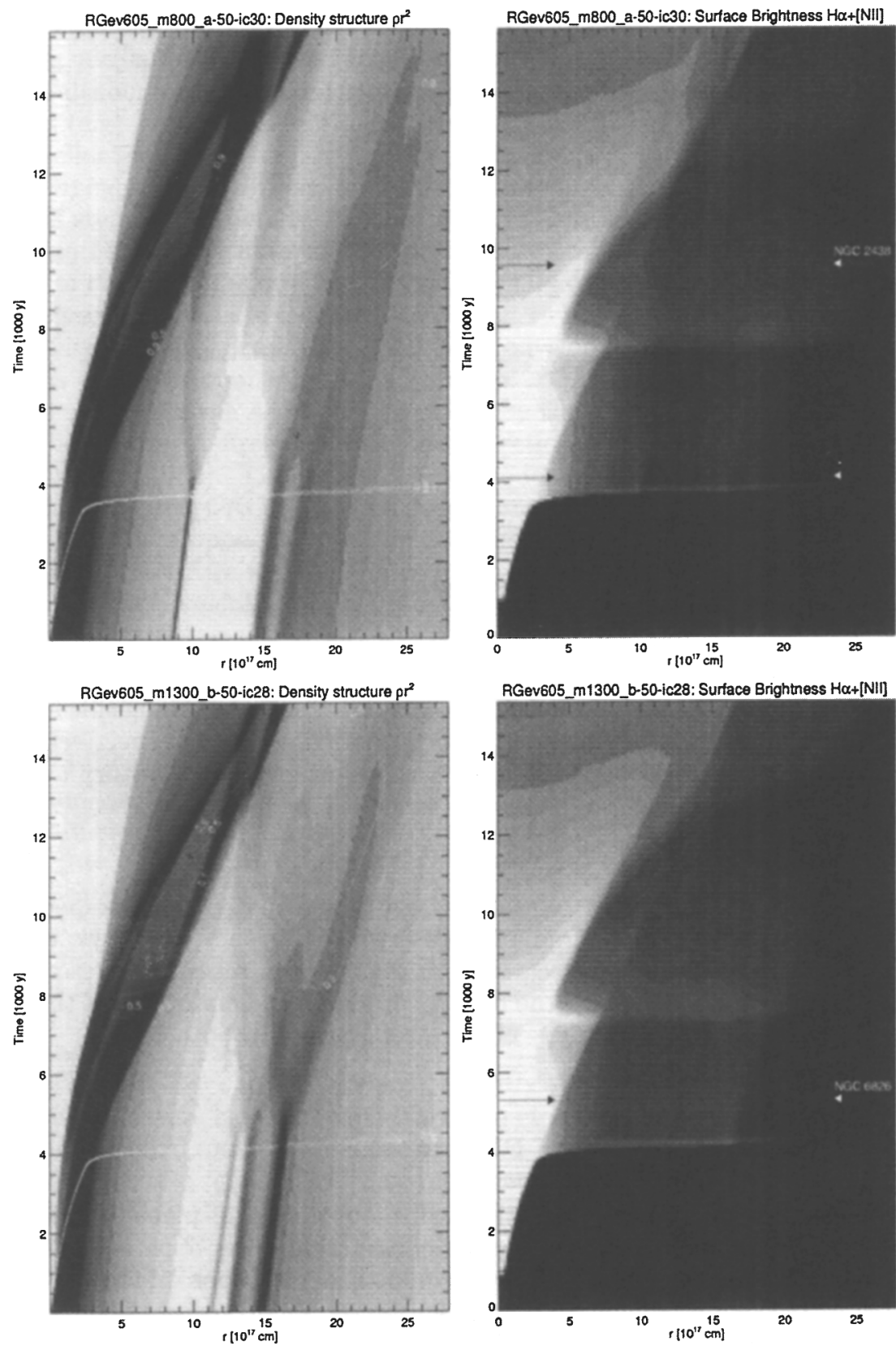

Figure 3. Left: Temporal evolution of the radial density distribution of the circumstellar envelope during the $\mathrm{PN}$ phase of evolution, starting at $t=0$ from the O-rich (top) and C-rich (bottom) configurations shown in Fig. 2. Greyscales represent $\rho r^{2}$ (high densities are black), contours indicate the total ionization fraction $(0.1,0.3,0.5,0.7,0.9)$. Right: Corresponding evolution of the surface brightness in $\mathrm{H}_{\alpha}+[\mathrm{NII}]$. The variation of $\log I$ is shown in the range 0 (white) to -4 (black). 
ionization, recombination, heating and cooling of due to $\mathrm{H}, \mathrm{He}, \mathrm{C}, \mathrm{N}, \mathrm{O}, \mathrm{Ne}$ with all of their ionization stages (Marten \& Szczerba 1997).

Starting at the tip of the AGB with the configurations shown in Fig. 2, we display in Fig. 3 the temporal evolution of the density structure and surface brightness profile $\left(\mathrm{H}_{\alpha}+[\mathrm{NII}]\right)$ over a time interval of about 15000 years. As far as the visibility of haloes is concerned, the basic evolutionary phases that can be distinguished according to our radiation hydrodynamics simulations are briefly described in the following.

Within 1000 years, the inner regions of the circumstellar matter become ionized and form a young, compact single-shell PN. As the effective temperature and wind power of the central star increase, the PN evolves into a typical doubleshell nebula. As may be seen from Fig. 3, the nebula remains optically thick and is ionization bounded for about 3500 years. During this period of time, the sharp density features created on the AGB are well preserved, but no halo can be seen because the extended AGB envelope is still neutral.

When the ionization front finally breaks through (horizontal contour lines near $t=4000$ years), the outer regions are ionized within a few 100 years, and become visible as a real AGB halo. We notice that the whole halo initially experiences a '[NII]-flash', lighting up strongly in [NII] for a very short time $(<100 \mathrm{yrs})$ when ionization first changes from NI to NII, before reaching the main ionization stage NIII (see bright horizontal feature in the lower part of right panels of Fig. 3). For times $t=4120$ and 5300 years (arrows), the surface brightness profiles are shown in Fig. 1 of Corradi et al. (this volume). In the oxygen-rich sequence, we can see a double halo, where the edges of the two haloes correspond to the inner and outer position of the density 'gap'. In the carbonrich case, we see one extended AGB halo, whose surface brightness profile is very similar to that of NGC 6826 . Here the bright limb of the halo is related to the density peak which was produced by the last thermal pulse on the AGB.

As soon as the halo is fully ionized, it begins to change dynamically. As temperature and pressure increase, considerable velocity fields build up at the positions of strong density gradients, eliminating any thin AGB shells and smoothing out the sharp edges of the density 'gaps'. Local flow velocities in the halo are found to range between -5 and $30 \mathrm{~km} / \mathrm{s}$. Such velocity fields are probably hard to observe spectroscopically due to averaging along the line of sight.

After roughly 7500 years, the stellar luminosity has dropped below $L=$ $1000 \mathrm{~L}_{\odot}$, and the plasma in the shell and the halo begins to recombine partially. The halo becomes distinctly brighter as NIII recombines to NII. The nebular shell, however, decreases in brightness and forms a recombination halo. The oxygen-rich sequence provides a good model for NGC 2428 at $t \approx 9600$ yrs (Corradi et al. 2000). Note that by this time the outer (AGB) halo has changed from what was formerly a 'double halo' to a single halo of intermediate size. The outer edge of this new halo is defined by the density maximum which has formed in the center of the initial density 'gap' as a result of the dynamical evolution of the ionized AGB halo.

As the nebula expands further, it re-ionizes, even though the stellar luminosity continues to decrease. According to our models, a diffuse halo might still be visible during these late evolutionary stages. 


\section{Conclusions}

Guided by recent numerical simulations, we conclude that the sharp, limbbrightened edges of young $A G B$ haloes are probably not the result of AGB wind interaction with the interstellar medium, but rather a signature of the density structure formed through mass loss variations and two-wind interaction on the AGB. Once ionized, however, the imprinted AGB density structure begins to evolve dynamically, resulting in a loss of information about the AGB mass loss history. Old AGB haloes may have produced their very own density profiles and are therefore not recommended for AGB wind paleontology studies.

\section{References}

Arndt, T.U., Fleischer, A.J., \& Sedlmayr, E. 1997, A\&A, 327, 614

Balick, B. 1987, AJ, 94, 671

Balick, B., Gonzalez, G., \& Frank, A. 1992, ApJ, 392, 582

Blöcker, T. 1995, A\&A, 297, 727

Chu, Y.-H., Jacoby, G. H., \& Arendt, R. 1987, ApJS, 64, 529

Corradi, R. L. M., Schönberner, D., Steffen, M., \& Perinotto, M. 2000, A\&A, 354, 1071

Duncan, J.C. 1937, ApJ, 86, 496

Frank, A. 1994, AJ, 107, 261

Haijan, A.R., Frank, A., Balick, B., \& Terzian, Y. 1997, ApJ, 477, 226

Marten, H., \& Schönberner, D., 1991, A\&A, 248, 590

Marten, H., \& Szczerba, R. 1997, A\&A, 325, 1132

Mellema, G. 1994, A\&A, 290, 915

Olofsson, H., Bergman, P., Lucas, R., et al. 2000, A\&A, 353, 583

Plait, P., \& Soker, N. 1990, AJ, 99, 1883

Pauldrach, A., Puls, J., Kudritzki, R.-P., et al. 1988, A\&A, 207, 123

Rauch, T., Köppen, J., Napiwotzki, R., \& Werner, K. 1999, A\&A, 347, 169

Schönberner, D., Steffen, M., Stahlberg, J., et al. 1997, in Advances in Stellar Evolution, R.T. Rood \& A. Renzini, Cambridge: University Press, 146

Simis, Y.J.W., Icke, V., \& Dominik, C. 2001, A\&A, 371, 205

Steffen, M., Szczerba, R., \& Schönberner, D. 1998, A\&A, 337, 149

Steffen, M., \& Schönberner, D. 2000, A\&A, 357, 180

Steffen, M., \& Schönberner, D. 2001, in Post-AGB objects as a phase of stellar evolution, eds. R. Szczerba \& S.K. Gorny, Kluwer, p. 131

Trimble, V., \& Sackmann, I.-J. 1978, MNRAS, 182, 97

Tylenda, R. 1986, A\&A, 156,217

Vassiliadis, E., \& Wood, P.R. 1992, Proc. Astron. Soc. Austr., 10 (1), 30

Vassiliadis, E., \& Wood, P.R. 1993, ApJ, 413, 641

Winters, J.M., Le Bertre, T., Jeong, K.S., et al. 2000, A\&A, 361, 641 